\title{
A NOTE ON THE KAKEYA MAXIMAL OPERATOR
}

\author{
Jose A. BARrionuevo
}

\begin{abstract}
In this paper we obtain an upper bound for the $L^{2}$ norm for maximal operators associated to arbitrary finite sets of directions in $\mathbb{R}^{2}$.
\end{abstract}

\section{Introduction}

Let $\Omega_{N}$ denote a collection of $N$ unit vectors in $\mathbb{R}^{n}$. The Kakeya maximal operator $M_{\Omega_{N}}$ is defined on locally integrable functions $f$ on $\mathbb{R}^{n}$ as

$$
M_{\Omega_{N}} f(x)=\sup _{x \in R \in \mathcal{B}_{\Omega}} \frac{1}{|R|} \int_{R}|f(y)| d y,
$$

where $\mathcal{B}_{\Omega}$ denotes the class of all rectangles with longest side parallel to some $\omega$ in $\Omega_{N}$, and where $|A|$ represents the Lebesgue measure of $A$.

It is conjectured that $M_{\Omega_{N}}$ satisfies the following estimate:

$$
\left\|M_{\Omega_{N}} f\right\|_{L^{n}\left(\mathbb{R}^{n}\right)} \leq C_{n} \log N\|f\|_{L^{n}\left(\mathbb{R}^{n}\right)},
$$

where $C_{n}$ is independent of $f$ and $\Omega_{N}$.

This estimate was proved for the case of radial functions by Carbery, Hernández and Soria in [CHS]. For $n=2$ the cases of uniformly distributed directions and of a lacunary sequence have been solved for some time. We refer to Stein's book [St] and references therein (see also Wolff [W]) for much more about related maximal functions and its applications to other problems in harmonic analysis. For dimensions higher than two, the lacunary case was established by Carbery in [Ca] and the case of uniformly distributed directions along a curve of finite type was proved by the author in [B2].

In this paper we prove the following partial result for dimension 2 .

Theorem 1. There exists a constant $C$ such that for every $f$ in $L^{2}\left(\mathbb{R}^{2}\right)$ one has

$$
\left\|M_{\Omega_{N}} f\right\|_{L^{2}\left(\mathbb{R}^{2}\right)} \leq C N^{\epsilon_{N}}\|f\|_{L^{2}\left(\mathbb{R}^{2}\right)},
$$

where $\epsilon_{N}=2 / \sqrt{\log N}$ and $C$ is an absolute constant.

Received June 13, 1995. 
For every $N$, define

$$
\Phi_{N}=\sup _{\Omega_{N}}\left\{\left\|M_{\Omega_{N}}\right\|\right\}
$$

where $\|S\|$ denotes the $L^{2}$-operator norm of $S$ and the sup is taken over all finite subsets of $S^{1}$ with $N$ elements. Clearly $\Phi_{N}$ is well defined since one has the obvious estimate $\Phi_{N} \leq C N$. Also, it is known from the uniformly distributed case that $\Phi_{N} \geq C \log N$.

In what follows $C$ will denote a constant not necessarily the same on each occurrence but which is independent of $N$ and $f$.

\section{Proof}

The proof of Theorem 1 is based on a variant of the " $T T^{\star}$ method". Earlier versions of the method are in Stein [St] p. 317 and references in there. Other variants were independently developed by Nevo [Nevo] and the author [B1] for different purposes.

For simplicity we will assume that $\Omega_{N}=\left\{\omega_{1}, \ldots, \omega_{N}\right\}$, where $\omega_{i}=$ $\left(1, y_{i}\right)$ with $0 \leq y_{1}<y_{2}<\cdots<y_{N} \leq 1$. These affect $M_{\Omega_{N}}$ only by a multiplicative constant. Also let $d_{i}=y_{i+1}-y_{i}$.

For $m=(k, i)$ where $k$ is an integer and $i$ belongs to $\{1, \ldots, N\}$ define

$$
\begin{aligned}
T_{m}^{\Omega_{N}} f(x) & =\frac{1}{2^{k+1}} \int_{-2^{k}}^{2^{k}} f\left(x-t \omega_{i}\right) d t \\
T^{\Omega_{N}} f(x) & =\sup _{m}\left|T_{m} f(x)\right| .
\end{aligned}
$$

Note that the for each $m, T_{m}^{\Omega_{N}}$ is a positive self-adjoint linear operator.

Then a simple geometric argument shows that for any non-negative $f$ one has:

$$
M_{\Omega_{N}} f(x) \leq C T^{\Omega_{N}} f(x),
$$

and we are left to prove

$$
\left\|T^{\Omega_{N}} f\right\|_{L^{2}} \leq C N^{\epsilon_{N}}\|f\|_{L^{2}},
$$

and since (3) is independent of $\Omega_{N} \subset S^{1}$ a similar estimate for $\Phi_{N}$ will follow.

The proof of (3) is based in the following pointwise estimate valid for $f \geq 0$ and $0<\alpha<1$ :

$$
\begin{gathered}
T_{m}^{\Omega_{N}} T_{n}^{\Omega_{N}} f(x) \leq \\
C\left(\left(T_{\beta(m)}^{\Omega_{N}} S_{n}^{\Omega_{N}^{1}}+T_{\beta(n)}^{\Omega_{N}} S_{m}^{\Omega_{N}^{1}}+\sum_{l=1}^{\left[N^{\alpha}\right]} T_{\varphi(m)}^{\Omega_{N}} H_{n}^{l}+T_{\varphi(n)}^{\Omega_{N}} H_{m}^{l}\right) f(x)\right),
\end{gathered}
$$


where:

- $\varphi: \Omega_{N} \rightarrow \Omega_{N}$ is given by $\varphi(k, i)=(k+1, i)$,

- $H_{n}^{l}$ are 1-dimensional averages in the $y$-direction, thus dominated by the one dimensional Hardy-Littlewood maximal operator acting in the $y$ - direction, and

- $S_{m}^{\Omega_{N}^{1}}=T_{\beta(m)}^{\Omega_{N}}$, where $\beta$, to be determined later, is a function with values on a subset $\Omega_{N}^{1} \subset \Omega_{N}$ with $\left[N^{1-\alpha}\right]$ elements. Here $[z]$ denotes the smallest integer greater than $z$. Hence

$$
\left\|\sup _{m} S_{m}^{\Omega_{N}^{1}}\right\|_{L^{2}} \leq \Phi_{\left[N^{1-\alpha}\right]}
$$

Assuming (4) for the moment one argues as in the proof of proposition 1.1 of [B1] obtaining

$$
\Phi_{N} \leq C\left(\left[N^{\alpha}\right]+\Phi_{\left[N^{1-\alpha}\right]}\right) \leq C \max \left\{N^{\alpha}, \Phi_{\left[N^{1-\alpha}\right]}\right\}
$$

Starting with the bound $\Phi_{N} \leq C N$ we repeatedly apply (5) with the appropriate value of $\alpha$ on each step. This gives:

$$
\begin{aligned}
\Phi_{N} \leq & C^{2} \max \left\{N^{\alpha}, N^{1-\alpha}\right\} \Rightarrow \Phi_{N} \leq C^{2} N^{\frac{1}{2}} \\
\Phi_{N} \leq & C^{3} \max \left\{N^{\alpha}, N^{\frac{1-\alpha}{2}}\right\} \Rightarrow \Phi_{N} \leq C^{3} N^{\frac{1}{3}} \\
\vdots & \vdots \\
\Phi_{N} \leq & C^{p} \max \left\{N^{\alpha}, N^{\frac{1-\alpha}{p-1}}\right\} \Rightarrow \Phi_{N} \leq C^{p} N^{\frac{1}{p}} .
\end{aligned}
$$

The minimum of the right hand side of (6) is achieved for $p=\epsilon_{N}$. Thus, in order to prove the theorem we only need to establish (4).

The proof uses the following simple geometric fact whose proof is contained in [B1] Proposition 2.4:

Suppose $m=(i, k), n=(j, r), i>j$, and $k>r$. Then

$$
T_{m}^{\Omega_{N}} T_{n}^{\Omega_{N}} f(x) \leq C \frac{1}{|R|} \chi_{R} * f(x)
$$

where $R$ is a rectangle with dimensions $2^{i+1} \times 2^{j+1} \sin \theta$ with longest side parallel to $\omega_{k}, \theta$ is the angle between $\omega_{k}$ and $\omega_{r}$, and $\chi_{R}$ is the indicator function of $R$.

We divide the proof in two cases: 
a): $\omega_{k}$ "near" $\omega_{r}$ : that is, $\sin \theta \leq \Delta_{n}$, where

$$
\begin{aligned}
\Delta_{n}= & \max \left\{\delta_{1}^{n}, \delta_{2}^{n}, \ldots, \delta_{\left[N^{\alpha}\right]}^{n}\right\}, \text { and } \\
\delta_{1}^{n}= & d_{r}, \\
\delta_{2}^{n}= & \max \left\{2 \delta_{1}, d_{r}+d_{r+1}\right\}, \\
\vdots & \vdots \\
\delta_{\left[N^{\alpha}\right]}^{n}= & \max \left\{2 \delta_{\left[N^{\alpha}\right]-1}^{n}, d_{r}+\cdots+d_{r+\left[N^{\alpha}\right]}\right\} .
\end{aligned}
$$

This gives

$$
\frac{1}{|R|} \chi_{R} * f(x) \leq C \sum_{l=1}^{\left[N^{\alpha}\right]} T_{\varphi(m)}^{\Omega_{N}} H_{n}^{l} f(x),
$$

with

$$
H_{n}^{l} f(x)=\frac{1}{2^{j+1} \delta_{l}^{n}} \int_{-\delta_{l}^{n} 2^{j}}^{\delta_{l}^{n} 2^{j}} f(x-t(0,1)) d t,
$$

so that for each $l, \sup _{n} H_{n}^{l} f(x)$ is dominated by the one-dimensional Hardy-Littlewood operator.

b): $\omega_{k}$ "far" from $\omega_{r}$ : define $\beta(j, r)=\left(j+1,\left[s(r) N^{\alpha}\right]\right)$ where $s(r)$ is the smallest integer for which $\left[s(r) N^{\alpha}\right]>r$. In this case $\omega_{k}-\omega_{r} \approx \omega_{k}-\omega_{s N^{\alpha}}$ and this gives

$$
\frac{1}{|R|} \chi_{R} * f(x) \leq C T_{\beta(m)}^{\Omega_{N}} S_{n}^{\Omega_{N}^{1}} f(x),
$$

where $S_{n}^{\Omega_{N}^{1}} f(x)=T_{n}^{\Omega_{N}}$ restricted to a subset of $N^{1-\alpha}$ directions. Combining (8) and (9) with the appropriate symmetries in $m$ and $n$, gives (4). This completes the proof.

Remarks. It is possible to obtain a power of $N$ slightly better than $\epsilon_{N}$ in Theorem 1 by a more careful analysis of (4). We do not pursue this here in order to keep the presentation simple. On the other hand one can verify that in order to obtain the conjectured $\log N$ estimate by this method, one must improve (4).

\section{Acknowledgement}

This work is a continuation of the author's Ph.D. dissertation and he would like to thank his advisor, Prof. Allan Greenleaf, once again. 


\section{References}

[B1] J. Barrionuevo, $L^{2}$ Estimates for some Kakeya type maximal operators, Trans. Amer. Math. Soc. 335 (1993), 667-682.

[B2] _ Averages along uniformly distributed directions on a curve, Proc. Amer. Math. Soc. 119, 3 (1993), 823-827.

[Ca] A. Carbery, Differentiation in lacunary directions and an extension of the Marcinkiewicz multiplier theorem, Ann. Inst. Fourier, Grenoble, 38 (1988), 157-168.

[CHS] A. Carbery, E. Hernández, and F. Soria, Estimates for the Kakeya maximal operator on radial functions in $\mathbb{R}^{n}$, in Harmonic Analysis, edited by $\mathrm{S}$. Igari, Springer-Verlag (1991), 41-50.

[Nevo] A. Nevo, Harmonic Analysis and pointwise ergodic theorems for noncommuting transformations, J. Amer. Math. Soc., 7 (1994), 875-902.

[St] E. M. Stein, Harmonic Analysis: Real-Variable Methods, Orthogonality, and Oscillatory Integrals, Princeton Univ. Press, (1993).

[W] T. H. Wolff, An improved bound for Kakaya type maximal functions - to appear.

Department of Mathematics and Statistics, University of South Alabama

E-mail address: jose@mathstat.usouthal.edu 